\title{
Spin Current Cross-Correlations as a Probe of Magnon Coherence
}

\author{
Scott A. Bender, ${ }^{1}$ Akashdeep Kamra, ${ }^{2,3}$ Wolfgang Belzig, ${ }^{3}$ and Rembert A. Duine ${ }^{1,4,2}$ \\ ${ }^{1}$ Utrecht University, Princetonplein 5, 3584 CC Utrecht, Netherlands \\ ${ }^{2}$ Center for Quantum Spintronics, Department of Physics, Norwegian University of Science and Technology, \\ Trondheim, Norway \\ ${ }^{3}$ Department of Physics, University of Konstanz, D-78457 Konstanz, Germany \\ ${ }^{4}$ Department of Applied Physics, Eindhoven University of Technology, P.O. Box 513, 5600 MB Eindhoven, Netherlands
}

(Received 25 November 2018; published 6 May 2019)

\begin{abstract}
Motivated by the important role of the normalized second-order coherence function, often called $g^{(2)}$, in the field of quantum optics, we propose a method to determine magnon coherence in solid-state devices. Namely, we show that the cross-correlations of pure spin currents injected by a ferromagnet into two metal leads, normalized by their dc value, replicate the behavior of $g^{(2)}$ when magnons are driven far from equilibrium. We consider two scenarios: driving by ferromagnetic resonance, which leads to the coherent occupation of a single mode, and driving by heating of the magnons, which leads to an excess of incoherent magnons. We find an enhanced normalized cross-correlation in the latter case, thereby demonstrating bunching of nonequilibrium thermal magnons due to their bosonic statistics. Our results contribute to the burgeoning field of quantum magnonics, which seeks to explore and exploit the quantum nature of magnons.
\end{abstract}

DOI: 10.1103/PhysRevLett.122.187701

Introduction.-In the early years of quantum mechanics, the drive towards demonstrating its classical limit led Schrödinger to examine a class of wave functions that replicates the classical dynamics of a harmonic oscillator [1]. In the language of second quantization, these states turned out to represent the eigenstates of the annihilation operator [2]. These have come to be known as "coherent states". Remarkably, the purely quantum phenomenon of Bose-Einstein (BE) condensation results in a condensate characterized by a coherent state wave function, comprising phenomena like superconductivity and superfluidity.

Characterizing and quantifying the "quantumness" of photonic states is one of the central themes in the field of quantum optics $[3,4]$. A measure particularly relevant for condensation phenomena is the so-called normalized second-order temporal coherence function, typically denoted by $g^{(2)}(\tau)$ :

$$
g^{(2)}(\tau)=\frac{\langle: \hat{I}(t) \hat{I}(t+\tau):\rangle}{\langle\hat{I}(t)\rangle\langle\hat{I}(t+\tau)\rangle},
$$

where $\hat{I}$ is the optical intensity magnitude operator, : : represents normal ordering of the photon ladder operators, and \langle\rangle denotes the expectation value. For a coherent state (e.g., a laser), the numerator in Eq. (1) factorizes, and $g^{(2)}(\tau)=1 . g^{(2)}(\tau)$ also quantifies the relative probability of detecting two photons separated by a time lag $\tau$. Specifically, $g^{(2)}(0)$ gives the rate of simultaneous detection of two photons in a given optical state. A single-mode thermal light field exhibits the so-called photon bunching effect, corresponding to $g^{(2)}(0)=2$, which is indicative of the bosonic nature of the photons. The concept of bosonic bunching has been extended beyond quantum optics in more recent years; an analogue of $g^{(2)}(\tau)$ has been measured in a system of ultracold trapped atoms, demonstrating temporal atomic bunching and $\mathrm{BE}$ condensate coherence [5].

While quantum optics is now a mature field, similar advances have just begun to be made for bosonic excitations in magnets-magnons [6,7]. Recent advances [8] in manipulating and detecting these excitations have enabled exciting fundamental physics in magnetic systems with the potential for technological applications [9]. Observation of a nonequilibrium magnon $\mathrm{BE}$ condensate has been reported $[6,10]$ wherein magnon coherence has been demonstrated by various optical techniques [11]. The same system seems to exhibit a superfluidlike flow of spin current providing further evidence towards these claims [12], which, however, are not uncontested [13] but are recently supported by new observations [14]. The existence of robust spin superfluidity with a different physical origin has been postulated in specific circumstances [15-18]. However, a direct signature of magnon coherence measurable in all-solidstate systems, where optical probes may not be feasible, is still lacking.

In this Letter, we give a proposal for the solid-state detection of magnon state coherence, adapting concepts from quantum optics to metal-magnet hybrid structures. We show that metallic contacts, such as those used in nonlocal magnon transport experiments [19], can play the role of coherence-sensitive "detectors" of magnon 


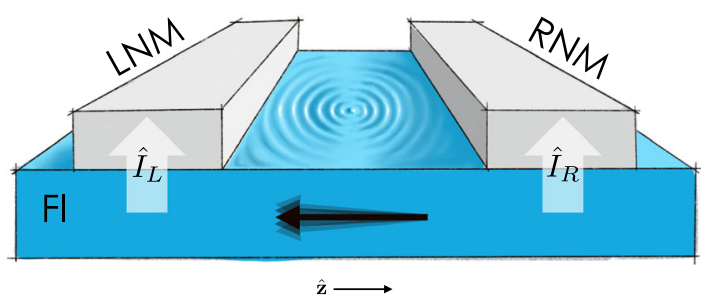

FIG. 1. Magnetic heterostucture, with ferromagnetic insulator (FI) and metallic leads (LNM and RNM). The arrow in the FI depicts its total spin. Spin currents $\hat{I}_{L}$ and $\hat{I}_{R}$ are injected into the LNM and RNM, respectively, when the FI is excited.

emission, in analogy with the photon detectors in quantum optical experiments. A key difference between quantum optical and spintronic systems lies in the fact that light, due to its long wavelength, weak interaction with surroundings, and ability to propagate in vacuum, exhibits coherence over large distances. This eliminates the need for the explicit inclusion of detectors in its description. In solidstate systems, we find it prudent to develop the coherence theory of magnons for a specific realization of detection integrated into the device structure. Nevertheless, the ideas developed herein are general and can be adapted to a different detection scheme. Furthermore, our findings are suggestive of employing heat currents as a probe into phonon coherences in an analogous manner.

Normalized spin current cross-correlation.-We consider a ferromagnetic insulator (FI) in contact with two nonmagnetic left and right metal (LNM and RNM) leads (e.g., see Fig. 1), into which spin current is injected and can be measured via the inverse spin Hall effect [8]. We define the normalized spin current cross-correlation $c^{(2)}(\tau)$ :

$$
c^{(2)}(\tau) \equiv \frac{\frac{1}{2}\left\langle\left\{\hat{I}_{L}(t), \hat{I}_{R}(t+\tau)\right\}\right\rangle}{\left\langle\hat{I}_{L}(t)\right\rangle\left\langle\hat{I}_{R}(t+\tau)\right\rangle},
$$

where $\hat{I}_{l}$ with $l=L, R$ are operators corresponding to the spin currents injected into LNM and RNM, respectively, and $\{\cdots\}$ denotes an anticommutator. A key feature of this measure, distinguishing it from $g^{(2)}(\tau)$, is that the spin currents may be positive or negative. In this sense, LNM and RNM at finite temperatures may be considered as "nonideal detectors" which may also emit magnons back into the FI, in addition to absorbing (detecting) them from it. The optical detectors, in contrast, are designed to predominantly absorb, and hence detect, photons. Thus, the positivity of intensity in Eq. (1) is maintained in this case.

In the present work, we evaluate $c^{(2)} \equiv c^{(2)}(0)$ considering a single magnon mode driven into (i) a coherent state and (ii) a thermal state with temperature higher than the metal leads. We find that at sufficiently large drives, $c^{(2)}$ emulates the behavior expected from $g^{(2)} \equiv g^{(2)}(0)$ (Fig. 2), i.e., it approaches 1 and 2 for cases (i) and (ii) respectively, demonstrating it to be a valid coherence measure. The need for strong driving of the FI arises because magnon detection via the metal leads is rendered nonideal by the processes in which the metal leads emit magnons into the FI. At sufficiently large drives, quantified by the temperature of the metal leads, magnon absorption by the metal lead detectors dominates over emission, and the detection process is efficient and similar to its optical counterpart.

Electron-magnet coupling.-We consider the FI to be axially symmetric around the $z$ direction. Accordingly, we take the equilibrium macrospin to be oriented in the $-\mathbf{z}$ direction, so that excitations thereof carry spin in the $+\mathbf{z}$ direction. For simplicity, in order to contrast coherent and incoherent spin excitations of the FI, we allow for small angle dynamics of the macrospin $\mathbf{S}$ only, neglecting micromagnetic degrees of freedom. Such excitations are conveniently parametrized by the Holstein-Primakoff transformation:

$\hat{S}_{z} \equiv \hat{\phi}^{\dagger} \hat{\phi}-S, \quad \hat{S}_{-} \equiv \hat{S}_{x}-i \hat{S}_{y}=\sqrt{2 S-\hat{\phi}^{\dagger} \hat{\phi}} \hat{\phi}$,

where $S$ is the integer macrospin of the FI, and $\hat{\phi}$ and $\hat{\phi}^{\dagger}$ are, respectively, magnon annihilation and creation operators, subject to the bosonic commutation relations $\left[\hat{\phi}, \hat{\phi}^{\dagger}\right]=1$. The operator $\hat{\phi}$ has two components:

$$
\hat{\phi}=\hat{\varphi}+\varphi .
$$

The first term, $\hat{\varphi}$, corresponds to incoherent fluctuations, with $\langle\hat{\varphi}\rangle \equiv 0$, while the second, $\varphi$, is a $c$-number corresponding to a coherent magnon. Only $\varphi$ contributes to the ensemble-averaged transverse dynamics. Assuming small amplitudes for $\varphi$ and $\hat{\varphi}$, we obtain $\left\langle S_{x}\right\rangle=\sqrt{2 S} \operatorname{Re}[\varphi]$ and $\left\langle S_{y}\right\rangle=-\sqrt{2 S} \operatorname{Im}[\varphi]$.

The evolution of the heterostructure spin dynamics is governed by the Hamiltonian $\mathcal{H}=\mathcal{H}_{m}+\mathcal{H}_{e}+\mathcal{H}_{J}$. The uncoupled FI magnon Hamiltonian is given by $\mathcal{H}_{m}=E_{m} \hat{\phi}^{\dagger} \hat{\phi}$, with $E_{m}$ as the magnon gap or, equivalently, $\hbar$ times the ferromagnetic resonance frequency. The uncoupled normal-metal electron Hamiltonian is $\mathcal{H}_{e}=$ $\sum_{l=L, R} \mathcal{H}_{l}$, where $\mathcal{H}_{l}=\sum_{k \sigma} \epsilon_{k} \hat{b}_{l k \sigma}^{\dagger} \hat{b}_{l k \sigma}$, with $\hat{b}_{l k \sigma}$ as a $\sigma$-spin annihilation operator for an electron in the $l=L, R$ lead with quantum number $k$ [20]. Magnons in the FI and the spins of electrons in the normal metals are coupled by exchange at the metal-magnet interfaces, which is captured by hopping of spin between magnons and electron-hole excitations in the normal metals: $\mathcal{H}_{J}=$ $\sum_{K} J_{K} \hat{\phi}^{\dagger}\left(\hat{L}_{K}+\hat{R}_{K}\right)+$ H.c. [21]. Here $J_{K}$ is the effective exchange interaction for both interfaces, while $\hat{L}_{K}^{\dagger}=$ $\hat{b}_{L k \uparrow}^{\dagger} \hat{b}_{L \tilde{k} \downarrow}$ and $\hat{R}_{K}^{\dagger}=\hat{b}_{R k \uparrow}^{\dagger} \hat{b}_{R \tilde{k} \downarrow}$ are creation operators for up-electron-down-hole pairs in the left and right leads, respectively, with $K=k, \tilde{k}$ as a collective index. 
The coupling $\mathcal{H}_{J}$ gives rise to spin currents through the left and right interfaces. The operator expression for each of these currents is obtained from the Heisenberg equation of motion for the electron spin density $\hat{s}_{l}=\sum_{K}\left(\hat{b}_{l k \uparrow}^{\dagger} \hat{b}_{l k \uparrow}-\right.$ $\left.\hat{b}_{l k \downarrow}^{\dagger} \hat{b}_{l k \downarrow}\right)$ in the respective lead. The current in the left lead is

$$
\hat{I}_{L}=\frac{i}{2}\left[\hat{\mathcal{H}}_{J}, \hat{s}_{l}\right]=\sum_{K}\left(\hat{L}_{K}^{\dagger}-\hat{L}_{K}\right) .
$$

Similarly, the current in the right lead is $\hat{I}_{R}=\sum_{K}\left(\hat{R}_{K}^{\dagger}-\hat{R}_{K}\right)$. In equilibrium, the average currents $I_{L}=\left\langle\hat{I}_{L}\right\rangle$ and $I_{R}=\left\langle\hat{I}_{R}\right\rangle$ vanish. The exchange Hamiltonian also gives rise to correlations between LNM and RNM electrons. To zeroth order in $\hat{\mathcal{H}}_{J}$, such correlations are destroyed by coupling to the environment, and the equilibrium density averages are unaffected by the exchange coupling. Thus, for LNM and RNM electrons in equilibrium, $\left\langle\hat{b}_{l k \sigma}^{\dagger} \hat{b}_{l^{\prime} k^{\prime} \sigma^{\prime}}\right\rangle=$ $\delta_{l l^{\prime}} \delta_{k k^{\prime}} \delta_{\sigma \sigma^{\prime}} n_{F}\left(\epsilon_{k}\right)$ where $n_{F}(\epsilon)=1 /\left(1+e^{\left(\epsilon-\epsilon_{F}\right) / T}\right)$ is the Fermi-Dirac distribution with common electronic temperature $T$ and Fermi energy $\epsilon_{F} \gg T$. As phase coherent dynamics vanish in equilibrium, the $z$ component of the incoherent spin density under equilibrium conditions is $\left\langle\hat{\varphi}^{\dagger} \hat{\varphi}\right\rangle=N=N_{i}$, with $N_{i}=1 /\left(e^{E_{m} / T_{m}}-1\right)$ as the Bose-Einstein distribution for magnon energy $E_{m}$ and temperature $T_{m}$.

Driving the magnet.-We assume the normal metals to be ideal heat and spin sinks, so that the electrons there are described by equilibrium conditions discussed above. We consider two methods of directly driving the FI. First, ferromagnetic resonance (FMR) can coherently excite magnetic dynamics. Under the influence of a microwave field with frequency $\omega$, a coherent excitation described by $\varphi(t)=\varphi e^{i(\omega t+\theta)}$ is created. Here the U(1) symmetry of the FI is explicitly broken, as the precessional phase $\omega t+\theta$ is determined by the applied microwave. As a second driving scheme, incoherent magnetic dynamics can be excited beyond equilibrium by heating the FI to a temperature $T_{m}$ higher than that of the metal leads, for example by heating with a laser pulse. Thus, in the presence of one or both types of drives, the nonequilibrium magnon density, to zeroth order in $\hat{H}_{J}$ becomes $\left\langle\hat{\varphi}^{\dagger} \hat{\varphi}\right\rangle=N=N_{c}+N_{i}$, where $N_{c}=\varphi^{*} \varphi$. The steady-state current resulting from either type of drive has been calculated to second order in the exchange coefficient $J_{K}$ [21]:

$$
\left\langle\hat{I}_{L}\right\rangle=\left\langle\hat{I}_{R}\right\rangle=I_{L}=I_{R}=2 \pi D^{2} J^{2} E_{m}\left(N-N_{\mathrm{NM}}\right),
$$

where $N_{\mathrm{NM}}=1 /\left(e^{E_{m} / T}-1\right)$ is the Bose-Einstein distribution function describing electron-hole excitations in the leads. The Fermi-surface averaged square of the exchange interaction is given by $J^{2} \equiv \sum_{K}\left|J_{K}\right|^{2} \delta\left(\epsilon_{k}-\epsilon_{F}\right) \times$ $\delta\left(\epsilon_{\tilde{k}}-\epsilon_{F}\right) / D^{2}$, with $D$ as the electronic density of states at the Fermi energy $\epsilon_{F}$ in the metal leads. Thus we see that it is not possible to distinguish the coherent and incoherent magnons from the spin currents $I_{L}$ and $I_{R}$ alone, since these depend on the total number of magnons $N=N_{c}+N_{i}$. Instead, we turn to the cross-correlations of the spin currents.

Spin current cross-correlations.-We now investigate the current-current cross-correlator $C(\tau)=\frac{1}{2}\left\langle\left\{\hat{I}_{L}(t), \hat{I}_{R}(t+\tau)\right\}\right\rangle$ to obtain $c^{(2)}(\tau)$ in Eq. (2). One can see directly how $C(\tau)$ encodes information about magnon fluctuations. When the magnons are completely coherent $(\hat{\phi}=\varphi)$ and magnon fluctuations can be neglected, the coupling Hamiltonian $\mathcal{H}_{J}$ does not give rise to correlations between left and right electrons. Here, $C(\tau)=\left\langle\hat{I}_{L}(t)\right\rangle\left\langle\hat{I}_{R}(t+\tau)\right\rangle$ factorizes, yielding $c^{(2)}(\tau)=1$. If, however, incoherent fluctuations of the magnon operators are taken into account, then cross-correlations give rise to

$$
c^{(2)}(\tau)=1+\Delta c(\tau),
$$

where the correction $\Delta c(\tau)$ comes from the Wick decomposition of magnon correlators. As discussed above, we focus on equal-time $(\tau=0)$ correlations in the steady state, abbreviating $C \equiv C(0)$ and $\Delta c(0) \equiv \Delta c$.

The current-current cross-correlation function is calculated perturbatively in $J$ [22]. Such an approach is consistent with the idea that the metallic leads act as weak probes of the magnet, thereby preserving a well-defined notion of a magnon rather than an entangled magnonelectron-hole excitation. Relegating the detailed calculation to the Supplemental Material [24], we summarize our results here.

Because the lowest nonvanishing contribution to $\mathcal{C}$ is fourth order in $J$, the lowest order terms (which we consider here) each contain four magnon operators, or two factors of the magnon density. There are therefore three types of terms: those $\sim N_{c}^{2}$, those $\sim N_{i}^{2}$, and those $\sim N_{i} N_{c}$, which we denote as $C_{c c}, C_{i i}$, and $C_{i c}$, respectively. One thus has

$$
C=C_{c c}+C_{i i}+C_{i c}
$$

where $C_{i i}=C_{i i}^{(\times)}+C_{i i}^{(\|)}$and $C_{i c}=C_{i c}^{(\times)}+C_{i c}^{(\|)}$, with the superscripts $(x)$ and $(\|)$ respectively denoting diagrams that cross and do not cross the FI (see Supplemental Material [24]). As shown in the Supplemental Material [24], one finds $C_{c c}=I_{L}^{(c)} I_{R}^{(c)}, C_{i i}^{(\|)}=I_{L}^{(i)} I_{R}^{(i)}$, and $C_{i c}^{(\|)}=$ $I_{L}^{(c)} I_{R}^{(i)}+I_{L}^{(i)} I_{R}^{(c)}$, where $I_{l}^{(i)}=2 \pi D^{2} J^{2} E_{m}\left(N_{i}-N_{\mathrm{NM}}\right)$ and $I_{l}^{(c)}=2 \pi D^{2} J^{2} E_{m} N_{c}$ are the incoherent and coherent contributions to the $l=L, R$ spin current $I_{l}=I_{l}^{(i)}+I_{l}^{(c)}$. The sum of $C_{c c}$ and the uncrossed terms is thus equal to the product of the currents: $C_{c c}+C_{i i}^{(\|)}+C_{i c}^{(\|)}=I_{L} I_{R}$. One thus obtains $\Delta c=\left(C_{i i}^{(\times)}+C_{i c}^{(\times)}\right) / I_{L} I_{R}$, so that $c^{(2)}$ is obtained directly by evaluating the crossed diagrams. 
Next, let us consider the cross-correlations under various scenarios. First, in equilibrium, $N=N_{\mathrm{NM}}$, and the currents $I_{L}$ and $I_{R}$ vanish, as does coherent dynamics $(\varphi=0)$, so $C_{c c}=C_{i c}=0$. At finite temperature, however, incoherent magnons are excited by thermal fluctuations. While the uncrossed diagrams vanish $\left(C_{i i}^{(\|)}=I_{L} I_{R}=0\right)$, the crossed diagrams $C_{i i}^{(\times)}$do not, reflecting correlations between fluctuations of the left and right currents. Thus, in equilibrium, the normalized correlation coefficient $c^{(2)}=$ $1+\Delta c$ diverges.

Second, consider heating of the FI. Under heating, a spin current flows from the FI to the normal-metal leads. All of the coherent terms, which are $\propto N_{c}$, are zero, leaving $C=C_{i i}=C_{i i}^{(\times)}+C_{i i}^{(\|)}$. As can be seen in the left-hand side of Fig. 2, as $T_{m}$ increases from $T, I_{L}=I_{R}$ becomes nonzero, decreasing $c^{(2)}$ from infinity. As shown in the Supplemental Material [24], when $T_{m}$ is sufficiently large that $N_{m} \gg N$, one finds after some work that $C_{i i}^{(\times)} \rightarrow I_{L} I_{R}$, and therefore $c^{(2)} \rightarrow 2$. Under strong incoherent driving, thermal magnons dominate the spin currents and their correlations, and $c^{(2)} \sim\left\langle\hat{\varphi}^{\dagger} \hat{\varphi}^{\dagger} \hat{\varphi} \hat{\varphi}\right\rangle /\left|\hat{\varphi}^{\dagger} \hat{\varphi}\right|^{2}=2$, reflecting bunching of thermal magnons [25]. Note also that because of quantum fluctuations, even as the metal leads temperature $T$ is reduced to zero, a sufficiently large bias $\Delta T=$ $T_{m}-T$ is required in order to observe thermal magnon bunching, i.e., $c^{(2)}=2$.

Last, consider driving by FMR. Here, only coherent magnons are excited by the external field, and the spin currents $I_{L}=I_{L}^{(c)}=I_{R}=I_{R}^{(c)}$ are determined by the FMR power. Because the incoherent spin currents are zero $\left(N_{i}=N_{\mathrm{NM}}\right), C_{i i}^{(\|)}=C_{i c}^{(\|)}=0$, but the crossed terms $C_{i i}^{(\times)}$ and $C_{i c}^{(\times)}$survive, reflecting correlations that arise from spin cotunneling. At sufficiently large FMR power, however, $N_{c} \gg N_{i}$, and $C_{c c}=I_{L} I_{R} \gg C_{i i}^{(\times)}, C_{i c}^{(\times)}$, and therefore $\Delta c \rightarrow 0$, so $c^{(2)} \rightarrow 1$. Thus, at sufficiently large FMR power, heating

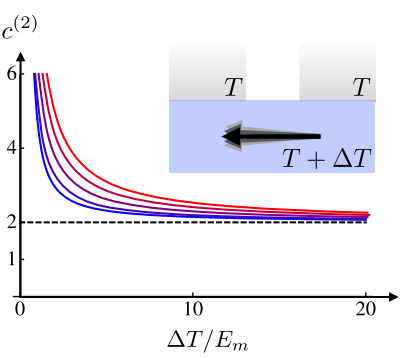

FMR

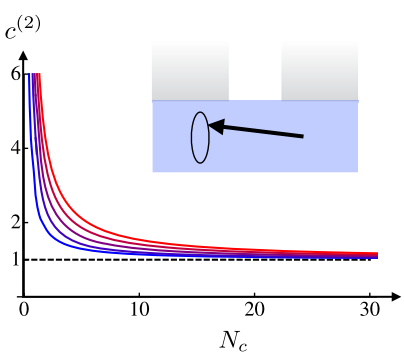

FIG. 2. Normalized spin-current cross-correlation $c^{(2)}$ for FI driven via heating (left) and FMR (right). The coherent mode number $N_{c}=(\chi h)^{2}$ is assumed to be driven by a circularly polarized FMR field $h$, with $\chi$ as the susceptibility. Solid lines, blue to red, represent electronic temperatures $T / E_{m}=0.01,2.5$, $5,7.5,10$. the spin current correlations are dominated by the coherent magnons, and $c^{(2)} \approx g^{(2)}=\left\langle\varphi^{*} \varphi^{*} \varphi \varphi\right\rangle /\left|\varphi^{*} \varphi\right|^{2}=1$. Note that even as $T \rightarrow 0, c^{(2)}$ is not necessarily equal to 1 at finite $N_{c}$. As with temperature biasing, quantum fluctuations of magnons and electron-hole pairs require that additionally $N_{c} \gg 1$ in order for $c^{(2)}$ to saturate at 1 .

Measurement of cross-correlations.-In the present device geometry, the spin current cross-correlation function $C$ can be obtained from the spin current fluctuations $\mathcal{S}(\tau) \equiv \frac{1}{2}\left\langle\left\{\delta \hat{I}_{L}(t), \delta \hat{I}_{R}(t+\tau)\right\}\right\rangle$, where $\delta \hat{I}_{l} \equiv \hat{I}_{l}-\left\langle\hat{I}_{l}\right\rangle$. In turn, $\mathcal{S}(\tau)$ is obtained by Fourier transforming the power spectral density $\mathcal{S}(\omega)$. It is straightforward to see that $C=I_{L} I_{R}+\mathcal{S}(\tau=0)$, so $\Delta c=\mathcal{S}(\tau=0) / I_{L} I_{R}$. It should be noted that in an actual measurement, the detector bandwidth sets the lower limit on $\tau$ for which $\mathcal{S}(\tau)$ can be measured. Thus, $\tau \rightarrow 0$ corresponds to $\tau$ approximately approaching the inverse of the detector bandwidth.

One might wonder why the spin current noise cannot alone be used to infer the coherence of the magnon state. The problem is that for both heating of the FI and FMR, $\mathcal{S}(0)$ increases with driving. In the former case, when the temperature difference between the FI and leads is large enough, $\mathcal{S}(0)=C_{i i}^{(\times)} \sim N_{i}^{2}$, while $I_{L} I_{R} \sim N_{i}^{2}$, so that their ratio $\Delta c$ saturates at constant value. In the latter case, the noise $\mathcal{S}(0)$ grows with $C_{i c}^{(\times)} \sim N_{c}$, but because $I_{L} I_{R} \sim N_{c}^{2}$, $\Delta c \sim 1 / N_{c}$ approaches zero with increasing FMR power. Thus, in order to distinguish the cross-correlations of coherent magnons from those of incoherent magnons, it is expedient to use the normalized quantity $c^{(2)}$, rather than simply the noise $\mathcal{S}$.

Discussion.-We have focused on a simple model to capture the essential physics of magnon bunching; we now comment on some of the approximations, along with their validity, employed in our analysis. First, we have neglected spatial dependence of cross-correlations on the assumption that the metal contacts are closer than the magnon coherence length (which for noninteracting magnons, is the thermal wavelength). If the contacts are further apart, a more general calculation, including finite wavelength thermal modes, should be carried out. By analogy with $g^{(2)}$, however, one may expect $c^{(2)}$ for thermal magnons to decay from 2 at distances smaller than the coherence length to 1 at larger distances; for coherent magnons, $c^{(2)}$ should remain equal to 1 [5], thus preventing one from distinguishing incoherent from coherent magnons if the contacts are not close enough. A distance-dependent study would, however, provide an avenue for measuring the magnon coherence length. If, on the other hand, the FI is too thin or the contacts too close, tunneling of electrons across the FI becomes possible, giving rise to a spin-polarized charge current that distorts the magnonic signal.

Second, the actual observation of pure spin currents and their fluctuations require conversion to measurable charge currents by, for example, the inverse spin Hall effect; this 
process may introduce additional charge current noise that is convoluted with that of cross-correlations. The conversion of spin to charge currents may also be complicated by interfacial spin-orbit coupling, which can spoil spin conservation; if interfacial spin-orbit coupling renormalizes effective transport coefficients and reduces the steady-state magnon efficiency [26], then in principle our dimensionless $c^{(2)}$ is unaffected. However, because in practice this could lead to a weaker signal and because inelastic spin-orbit scattering at the interface may convolute with the crosscorrelations, it would be prudent to focus on materials (e.g., yttrium-iron-garnet-platinum heterostructures) in which such effects are thought to be minimal [27].

Third, we have considered a macrospin model, wherein higher energy magnons are gapped out. While this may be a reasonable assumption for small structures at low temperatures, clearly as the FI is heated, micromagnetic modes must be taken into account. Last, we have neglected magnon-magnon interactions, which arise through additional, nonlinear terms in the magnon Hamiltonian. For a single magnon mode with contacts in close proximity, such terms serve only to renormalize the magnon gap and thus do not qualitatively change our results. When magnons are allowed to occupy a spectrum of modes with different energies, however, inelastic magnon scattering can facilitate interesting interplays between coherent and incoherent magnons [28], which might be observed via $c^{(2)}$.

We thank Asle Sudbø and Arne Brataas for valuable discussions. S. B. and R. D. are supported by funding from the Stichting voor Fundamenteel Onderzoek der Materie (FOM) and the European Research Council via Consolidator Grant No. 725509 SPINBEYOND. W. B. and A. K. are funded by the DFG through SFB 767 and the Alexander von Humboldt Foundation. R. D., A. K., and W. B. also acknowledge financial support from the Research Council of Norway through its Centers of Excellence funding scheme, Project No. 262633, "QuSpin".

[1] E. Schrödinger, Quantisierung als Eigenwertproblem, Ann. Phys. (Berlin) 384, 361 (1926); M. Martin Nieto, Displaced and squeezed number states, Phys. Lett. A 229, 135 (1997).

[2] R. J. Glauber, The quantum theory of optical coherence, Phys. Rev. 130, 2529 (1963); E. C. G. Sudarshan, Equivalence of Semiclassical and Quantum Mechanical Descriptions of Statistical Light Beams, Phys. Rev. Lett. 10, 277 (1963).

[3] D. F. Walls and G. J. Milburn, Quantum Optics (Springer, Berlin, 2008).

[4] C. Gerry and P. Knight, Introductory Quantum Optics (Cambridge University Press, Cambridge, England, 2004).

[5] V. Guarrera, P. Würtz, A. Ewerbeck, A. Vogler, G. Barontini, and H. Ott, Observation of Local Temporal Correlations in Trapped Quantum Gases, Phys. Rev. Lett. 107, 160403 (2011); M. Naraschewski and R. J. Glauber, Spatial coherence and density correlations of trapped Bose gases, Phys. Rev. A 59, 4595 (1999).

[6] G. E. W. Bauer, Eiji Saitoh, and B. J. van Wees, Spin caloritronics, Nat. Mater. 11, 391 (2012); V. V. Kruglyak, S. O. Demokritov, and D. Grundler, Magnonics, J. Phys. D 43, 264001 (2010); A. V. Chumak, V. I. Vasyuchka, A. A. Serga, and B. Hillebrands, Magnon spintronics, Magnon spintronics, Nat. Phys. 11, 453 (2015); E. B. Sonin, Spin currents and spin superfluidity, Adv. Phys. 59, 181 (2010).

[7] A. Kamra and W. Belzig, Super-Poissonian Shot Noise of Squeezed-Magnon Mediated Spin Transport, Phys. Rev. Lett. 116, 146601 (2016); A. Kamra, U. Agrawal, and W. Belzig, Noninteger-spin magnonic excitations in untextured magnets, Phys. Rev. B 96, 020411(R) (2017).

[8] J. E. Hirsch, Spin Hall Effect, Phys. Rev. Lett. 83, 1834 (1999); E. Saitoh, M. Ueda, H. Miyajima, and G. Tatara, Conversion of spin current into charge current at room temperature: Inverse spin-Hall effect, Appl. Phys. Lett. 88, 182509 (2006); S. O. Valenzuela and M. Tinkham, Electrical detection of spin currents: The spin-current induced Hall effect (invited), J. Appl. Phys. 101, 09 B103 (2007); Y. Kajiwara, K. Harii, S. Takahashi, J. Ohe, K. Uchida, M. Mizuguchi, H. Umezawa, H. Kawai, K. Ando, K. Takanashi, S. Maekawa, and E. Saitoh, Transmission of electrical signals by spin-wave interconversion in a magnetic insulator, Nature (London) 464, 262 (2010).

[9] I. Zutic, J. Fabian, and S. D. Sarma, Spintronics: Fundamentals and applications, Rev. Mod. Phys. 76, 323 (2004).

[10] S. O. Demokritov, V. E. Demidov, O. Dzyapko, G. A. Melkov, A. A. Serga, B. Hillebrands, and A. N. Slavin, Bose-Einstein condensation of quasi-equilibrium magnons at room temperature under pumping, Nature (London) $\mathbf{4 4 3 ,}$ 430 (2006).

[11] V. E. Demidov, O. Dzyapko, S. O. Demokritov, G. A. Melkov, and A.N. Slavin, Observation of Spontaneous Coherence in Bose-Einstein Condensate of Magnons, Phys. Rev. Lett. 100, 047205 (2008); M. Jäckl, V. I. Belotelov, I. A. Akimov, I. V. Savochkin, D. R. Yakovlev, A. K. Zvezdin, and M. Bayer, Magnon Accumulation by Clocked Laser Excitation as Source of Long-Range Spin Waves in Transparent Magnetic Films, Phys. Rev. X 7, 021009 (2017); P. NowikBoltyk, O. Dzyapko, V. E. Demidov, N. G. Berloff, and S. O. Demokritov, Spatially non-uniform ground state and quantized vortices in a two-component Bose-Einstein condensate of magnons, Sci. Rep. 2, 482 (2012); A. A. Serga, V. S. Tiberkevich, C. W. Sandweg, V. I. Vasyuchka, D. A. Bozhko, A. V. Chumak, T. Neumann, B. Obry, G. A. Melkov, A. N. Slavin, and B. Hillebrands, Bose-Einstein condensation in an ultra-hot gas of pumped magnons, Nat. Commun. 5, 3452 (2014); O. Dzyapko, P. Nowik-Boltyk, B. Koene, V. E. Demidov, J. Jersch, A. Kirilyuk, T. Rasing, and S. O. Demokritov, High-resolution magneto-optical Kerr-Effect spectroscopy of magnon Bose-Einstein condensate, IEEE Magn. Lett. 7, 1 (2016).

[12] D. A. Bozhko, A. A. Serga, P. Clausen, V. I. Vasyuchka, F. Heussner, G. A. Melkov, A. Pomyalov, V. S. L'vov, and B. Hillebrands, Supercurrent in a room-temperature BoseEinstein magnon condensate, Nat. Phys. 12, 1057 (2016).

[13] E. B. Sonin, Comment on "Supercurrent in a room temperature Bose-Einstein magnon condensate", arXiv:1607 
.04720v2; D. A. Bozhko, A. A. Serga, P. Clausen, V. I. Vasyuchka, G. A. Melkov, V. S. L'vov, and B. Hillebrands, On supercurrents in Bose-Einstein magnon condensates in YIG ferrimagnet, arXiv:1608.01813v2.

[14] D. A. Bozhko, J. E. Kreil, H. Y. Musiienko-Shmarova, A. A. Serga, V.S. L'vov, and B. Hillebrands, Long-distance supercurrent transport in a room-temperature Bose-Einstein magnon condensate, arXiv:1808.07407; A. J. E. Kreil, D. A. Bozhko, H. Y. Musiienko-Shmarova, V. I. Vasyuchka, V. S. L'vov, A. Pomyalov, B. Hillebrands, and A. A. Serga, From Kinetic Instability to Bose-Einstein Condensation and Magnon Supercurrents, Phys. Rev. Lett. 121, 077203 (2018).

[15] E. B. Sonin, Spin currents and spin superfluidity, Adv. Phys. 59, 181 (2010).

[16] H. Skarsvåg, C. Holmqvist, and A. Brataas, Spin Superfluidity and Long-Range Transport in Thin-Film Ferromagnets, Phys. Rev. Lett. 115, 237201 (2015).

[17] S. Takei and Y. Tserkovnyak, Superfluid Spin Transport Through Easy-Plane Ferromagnetic Insulators, Phys. Rev. Lett. 112, 227201 (2014).

[18] B. Flebus, S. A. Bender, Y. Tserkovnyak, and R. A. Duine, Two-Fluid Theory for Spin Superfluidity in Magnetic Insulators, Phys. Rev. Lett. 116, 117201 (2016).

[19] L. J. Cornelissen, J. Liu, R. A. Duine, J. Ben Youssef, and B. J. Van Wees, Long-distance transport of magnon spin information in a magnetic insulator at room temperature, Nat. Phys. 11, 1022 (2015); S. T. B. Goennenwein, R. Schlitz, M. Pernpeintner, K. Ganzhorn, M. Althammer, R. Gross, and H. Huebl, Non-local magnetoresistance in YIG/Pt nanostructures, Appl. Phys. Lett. 107, 172405 (2015).

[20] For simplicity, we assume identical leads, so that electrons in both leads have identical dispersions.

[21] S. A. Bender and Y. Tserkovnyak, Interfacial spin and heat transfer between metals and magnetic insulators, Phys. Rev. B 91, 140402(R) (2015).
[22] In nanojunctions in the ballistic transport regime, electron cross-correlations are directly related to the junction conductance by fluctuation dissipation because of charge conservation [23]. Such a relation applies to the magnonic spin current noise across one interface [7], but not to the cross-correlated spin current. Across the FI, however, we expect dephasing by magnon-phonon interactions to invalidate this relation, justifying a perturbative treatment where only the first few orders of $J$ are kept.

[23] A. Cottet, W. Belzig, and C. Bruder, Positive Cross Correlations in a Three-Terminal Quantum Dot with Ferromagnetic Contacts, Phys. Rev. Lett. 92, 206801 (2004); F. M. Souza, A. P. Jauho, and J. C. Egues, Spin-polarized current and shot noise in the presence of spin flip in a quantum dot via nonequilibrium Green's functions, Phys. Rev. B 78, 155303 (2008); Y. M. Blanter and M. Buttiker, Shot noise in mesoscopic conductors, Phys. Rep. 336, 1 (2000).

[24] See Supplemental Material at http://link.aps.org/ supplemental/10.1103/PhysRevLett.122.187701 for a diagrammatic calculation of $c^{(2)}$ up to lowest nonvanishing order in $J$.

[25] Note that under cooling of the FI, however, fluctuations of electron-hole excitations dominate over those of magnons; as the cross-correlations do not capture thermal bunching of electron-hole pairs, we do not find that $c^{(2)}$ saturates at 2 under cooling.

[26] K. Chen and S. Zhang, Spin Pumping in the Presence of Spin-Orbit Coupling, Phys. Rev. Lett. 114, 126602 (2015).

[27] X. Jia, K. Liu, K. Xia, and G. E. W. Bauer, Spin transfer torque on magnetic insulators, Europhys. Lett. 96, 17005 (2011).

[28] B. Flebus, P. Upadhyaya, R. A. Duine, and Y. Tserkovnyak, Local thermomagnonic torques in two-fluid spin dynamics, Phys. Rev. B 94, 214428 (2016); S. A. Bender and Y. Tserkovnyak, Thermally driven spin torques in layered magnetic insulators, Phys. Rev. B 93, 064418 (2016). 